\title{
Research on Image Restoration Algorithm for Dynamic Target
}

\author{
Hua Xiang \\ Xi'an Technological University, Weiyang District School Road, Xi'an, China \\ huaxiang79@126.com
}

\begin{abstract}
For movement target, image recognition with blur is a difficult problem to solve in image acquisition system. The paper proposes an image restoration algorithm based on inverse filtering and wiener filtering. According to original image of movement target by acquisition system, it analyses frequency spectrum characteristic, builds restoration model for image degradation, researches inverse filtering and wiener filtering technologies of image preprocessing algorithm and image restoration algorithm. Further it explains image quality assessment method, discusses objective assessment method without reference in detail. Dealing with the movement target image of actual acquisition, some images are processed based on the restoration method which proved. By contrasting algorithm with result, the restoration method is valid to eliminate blur and noise of disturbing influence from target moving, which proposed combining with inverse filtering and wiener filtering. The result shows that the edge characteristic is obvious and propitious to reflect original target actually.
\end{abstract}

Keywords: image restoration, blurring, inverse filtering, wiener filtering

\section{Introduction}

It is more and more important to obtain and utilize information in modem society, as well as image is the major of information source. Multifarious image is get in daily life, such as using camera to take photo of family life, using monitor to record sequence picture, using radar to search long distance object, and using infrared sensor camera to handle thermal picture, etc. During the period of imaging, copy, transfer, scan and display, it is unavoidable to be influenced by many degradation factors, like image blur and noise disturb, that greatly reduce the efficacy in application. Thus it needs to restore the blur image, which eliminate and weaken degradation effect and enhance both noisesignal ratio and resolution ratio [1-4]. The goal of image restoration is to renew original image by processing low quality image. It is the foundation of image process, pattern recognition and machine vision, which is significant research.

\section{Image Existing Problem of Dynamic Target}

Under the movement state, the reason of image's quality degradation is mainly the relative motion existing between the image collection device and the target, that the collected image is vague. This kind of phenomenon is common in our daily life, such as the target image taken by space shuttle, the high-speed car caught by traffic camera. During the process of taking, the circumstances like high relative speed or shaving with target would cause the image quality greatly reductively under the movement state [5].

The reduction of image quality is called image degradation, which form mainly contains image blurring, image distortion, image with noise and others. No matter in the way of optical, electronic or photoelectric, the image would degradate with different degree. For there are different ways to obtain image, the sort of 
degradation can be divided into different ways, like relative movement between target and camera, focal misalignment of camera, sensor noise, random atmospheric turbulence and phase differs in optical systems [6]. All these degradation factors would lead to image degradation or distortion.

Usually noise is a kind of unpredictable random signal, that it can only be learned by the means of statistics. Noise runs throughout the whole course of input, collection, process and output. The source of noise contains internal noise and external noise. External noise comes from antenna interference and electromagnetic waves interference into the system from the power line. Internal noise includes component noise (optical film grain noise, magnetic tapes limitation noise, CD defects noise, etc.), system circuit noise (secondary emission electronic noise in CRT circuit) and mechanical movement noise (current instability of joint vibration, shaking in magnetic tape, head and disk, etc)[7-10]. Therefore, denoise is an important step in image processing.

\section{Results and Analysis Image Restoration Algorithm}

\subsection{Frequency Spectrum Character Analysis under Movement State}

The image restoration of movement target means to eliminate degradation factors for degraded image to restore the way they are by the maximum similarity. As figure 1, the procedure of image degraduation can be modeled to a degraduation function and an addibive noise, which both effect the original image $f(x, y)$ and produce a degraduation image $g(x, y)$, get original image $\hat{f}(x, y)$ by using restoration filtering. Given $f(x, y)$, degradation factor $H$ and some priori knowledge of noise, then the degraduation model of image can be obtained.

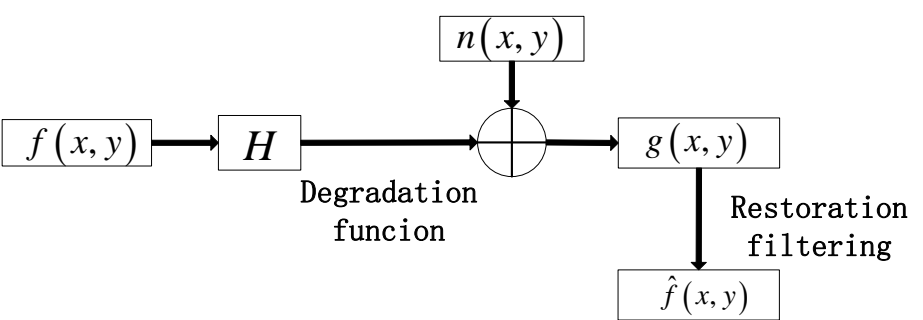

Figure 1. Image Restoration Model Diagram

The model can be formulated mathematics to

$$
g(x, y)=H[f(x, y)]+n(x, y)
$$

Define $\delta(x, y)$ is impulse function, $\delta(x-\alpha, y-\beta)$ is impulse function with delay. According to signals and systems knowledge, obviously $f(x, y)$ can be expressed to:

$$
f(x, y)=\int_{-\infty}^{\infty} \int_{-\infty}^{\infty} f(\alpha, \beta) \delta(x-\alpha, y-\beta) d \alpha d \beta
$$

Suppose $H$ is linear, put formula (2) into formula (1) to result in :

Order:

$$
g(x, y)=H\left[\int_{-\infty}^{\infty} \int_{-\infty}^{\infty} f(\alpha, \beta) \delta(x-\alpha, y-\beta) d \alpha d \beta\right]+n(x, y)
$$

$$
\begin{aligned}
& h(x, \alpha, y, \beta)=H \varpi \delta(x-\alpha, y-\beta), \text { then } \\
& g(x, y)=\int_{-\infty}^{\infty} \int_{-\infty}^{\infty} f(\alpha, \beta) h(x, \alpha, y, \beta) d \alpha d \beta+n(x, y)
\end{aligned}
$$


Thereinto $h(x, \alpha, y, \beta)$ is impulse response, in other word $h(x, \alpha, y, \beta)$ is the response in coordinate $\alpha, \beta$ by system $H$. Since in optics impluse is one optical point, $h(x, \alpha, y, \beta)$ is called point spread function (PSF) usually.

On the condition of space position constant, formular (4) would also be writen in formula (5).

$$
g(x, y)=\int_{-\infty}^{\infty} \int_{-\infty}^{\infty} f(\alpha, \beta) h(x-\alpha, y-\beta) d \alpha d \beta+n(x, y)
$$

From formula (5), it's seemed that the essence of relationship is convolution relationship between $f(x, y)$ and $h(x, y)$.

$$
g(x, y)=f(x, y) * * h(x, y)+n(x, y)
$$

Based on image analysis of frequency spectrum characteristic under the move state, it is discovered that the problem of vague and noisy interference exist from the collected images[11]. In order to obtain the realistic original image, it's should to recover degradation image. If only using inverse filter to recover image, noise disturb of restoration image is still very big, and the effect of image is still poor[12]. Therefore, the paper proposes a combined restoration algorithm with inverse filtering and wiener filtering based on the restored model for degraded image, that recovery image at best and solve the problem of image blur and noise interference.

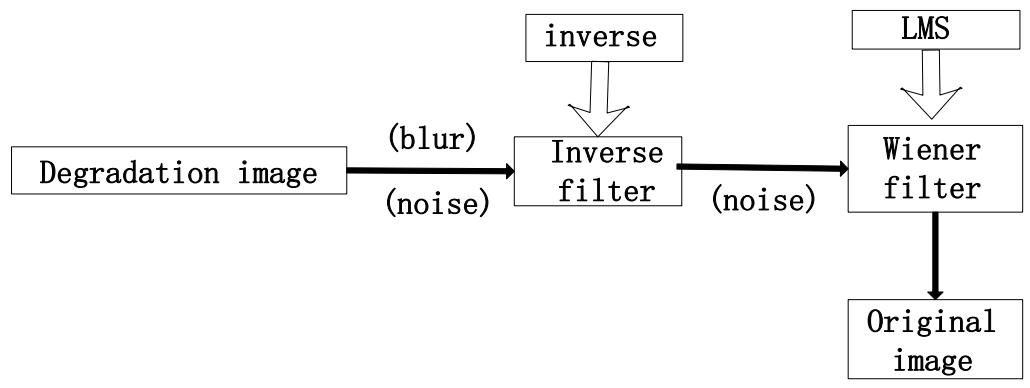

\section{Fugure 2. Restoration Algorithm Flow Combined Inverse Filtering and Wiener Filtering}

\subsection{Improved Restoration Algorithm of Inverse Filtering}

The procedure of inverse filter mainly includes[13]: first the original image is transform from space domain to Fourier frenqency domain, and make inverse filtering to degradation image, transform from Fourier frenqency domain to space domain, finally get the original image wantted. The formula (6) is transformed by Fourier, there is

$$
G(u, v)=H(u, v) F(u, v)+N(u, v)
$$

After transformed, there is

$$
F(u, v)=\frac{G(u, v)-N(u, v)}{H(u, v)}
$$

The formula (8) is made by Fourier inversion, there is:

$$
\hat{f}(x, y)=F^{-1}[F(u, v)]=F^{-1}\left\{\frac{G(u, v)-N(u, v)}{H(u, v)}\right\}
$$

In the formula (9), $\hat{f}(x, y)$ is the restoration image. 
From the formula above, there can be conducted that if the Fourier transform of degradation image $g(x, y)$ and point spread function $h(x, y)$ is known, the Fourier transform of restoration image can be derived by these two prior knowledges, and then the restoration image $\hat{f}(x, y)$ would be get by Fourier inversion, that's the principle of inverse filtering. If $N(u, v) / H(u, v)$ is very big, that means amplifing noise a lot, and the restoration image effect is not good. Actually the amplitude of $H(u, v)$ would decrease rapiddly along with origin distance in $u, v$ plane increasing, and the noise $N(u, v)$ would change slowly, when far away from origin distance in $u, v$ plane, the value of $N(u, v) / H(u, v)$ would become very big, which noise is amplified, the restoration can just carry in the range of frenquency domain.

Thereinto degeneracy factor $h(x, y)$ in movement blurry can be expressed as below:

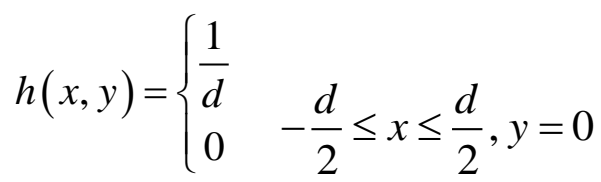

In the formula, $d$ indicates displacement in horizontal direction.

As inverse filtering algorithm is benefit in computation efficiency and easy to implement, it would choose suitable degradation operator according to the actual situation, the recovery image is clear, which the image vague problems is greatly improved. While for the image with loud noise, wiener filtering method is better to get restoration image.

Using the improved inverse filter algorithm, the problem can be solved for motion state of image vague, but noise is enlarged that causes noise interference is still big. So based on the improved inverse filter algorithm, it would add the improved wiener filtering algorithm to process of image noise problem.

\subsection{Improved Restoration Algorithm of Wiener Filtering}

In the process of image formation, transmission and storage, it is vulnerable to get noise interference. It is very important to lose image noisy during recovery. The image points disturbing by noise uniformly distribute between maximum and minimum values, which called Gaussian white noise. Aimed at the problem, the Wiener filter is adopted to image denoising[14].

The mean value of noise expresses the total strength in image. The mean value calculate formula as:

$$
\bar{n}=E[n(x, y)]=\frac{1}{M N} \sum_{x=1}^{M} \sum_{y=1}^{N} n(x, y)
$$

The variance formula of noise :

$$
e^{2}=E\left\{[n(x, y)-\bar{n}]^{2}\right\} \frac{1}{M N} \sum_{x=1}^{M} \sum_{y=1}^{N}[n(x, y)-\bar{n}]^{2}
$$

The noise $n(x, y)$ is Gaussian white noise of zero mean value, then

$$
e^{2}=\frac{1}{M N} \sum_{x, y}[g(x, y)-f(x, y) \square h(x, y)]^{2}
$$

In wiener filtering it's to searching for an estimate $\hat{f}$ to make statistical error function $e^{2}=E\left\{(f-\hat{f})^{2}\right\}$ least. The representation in frenquency domain indicates: 


$$
\hat{f}(u, v)=\left[\frac{1}{H(u, v)} \times \frac{|H(u, v)|^{2}}{|H(u, v)|^{2} \times s \frac{P_{n}(u, v)}{P_{f}(u, v)}}\right] G(u, v)
$$

Thereinto, $|H(u, v)|^{2}=H^{*}(u, v) H(u, v), G(u, v)$ is degenerate function, $H^{*}(u, v)$ indicates complex conjugate, $P_{n}(u, v)$ indicates noise power, $P_{f}(u, v)$ is power spectrum of original image. Formula (14) is wiener filtering, that the filter also called least mean square error filter or least square error filter. When noise-signal ratio of image is very big, $P_{n}(u, v)$ would far less than $P_{f}(u, v)$, then $P_{n}(u, v) / P_{f}(u, v)$ would approximate zero, here wiener filtering simplified as inverse filtering. While when noisesignal ratio of image is very small, $P_{n}(u, v)$ would far bigger than $P_{f}(u, v)$, then $P_{n}(u, v) / P_{f}(u, v)$ would approximate infinity, that wiener filtering avoids amplify problem of inverse filtering[15]. In the reality application, $P_{n}(u, v)$ and $P_{f}(u, v)$ are difficulty to compute to substitute for parameter $K$, as:

$$
\hat{f}(u, v)=\left[\frac{1}{H(u, v)} \times \frac{|H(u, v)|^{2}}{|H(u, v)|^{2} \times K}\right] G(u, v)
$$

The actualize steps of algorithm: make wiener filtering first time, estimate power spectrum $P_{f}(u, v)$ of original image and variance $e^{2}$ accoring to the first filtering result, and use formula (15) to second wiener filtering, here get the restoration image.

From that, the noise disturbing question of image can use wiener filtering to achieve image recovery, which the algorith essentially is to solve degenerate function $H(u, v)$ and noise-signal ratio $K$, that the accuracy choosen affect final restored effect. Wiener filtering is better to keep image edge and high frequency in detail. It's convenient to apply in estimate the filter parameter automaticly.

\section{Quality Assessment after Image Restored}

Image quality estimate includes subjective evaluation and objective evaluation. Due to uncertainty in the subjective evaluation, it is based on the private evaluation with the different way to observe the pros and cons. It is the most direct and common evaluation method adapting to human eye visual features. In order to get the recovery effect more accurate of image, it needs to evaluate image quality by the evaluation parameters [16].

The paper adopts no reference quality assessment method, that it can be evaluated without original image. But it's mostly aiming at a certain degradation sorts. It is general to evaluate image under the condition of no original image information.

\subsection{The Average Gray Gradient Method}

The average gray gradient method reflects the image contrast and texture characteristics. The method is taking subtracting, sum of squares and root mean square for adjacent pixel gray value in the $\mathrm{x}$ and $\mathrm{y}$ direction respectively. The greater the result is, the clearer the image is, and the quality the image is.

$$
G M G=\frac{1}{(M-1)(N-1)}
$$




$$
\sum_{i=1}^{M-1} \sum_{j=1}^{N-1} \sqrt{\frac{[f(i, j+1)-f(i, j)]^{2}+[f(i+1, j)-f(i, j)]^{2}}{2}}
$$

\subsection{Method of Laplacian}

The method of Laplacian is used in the neighborhood of $3 * 3$, by using Laplacian which each pixel get the 8 neighborhood differential value, and then work the sums of image sequence. The greater the value is, the clearer the image is, the brighter the outline is, and the better quality is.

$$
L S=\frac{\sum_{i=2}^{M-1} \sum_{j=2}^{N-1} \mid-f(i, j+1)-f(i+1, j-1)-f(i+1, j)-f(i+1, j+1)}{(M-2)(N-2)}
$$

With no reference quality estimate method, to evaluate the quality of restoration image, it represents better quality of the recovery image, when the value is bigger by calculate the image $G M G$ and $L S$ [17].

\section{Results and Analysis}

In order to verify whether the inverse filter or wiener filter can solve the problem of image blur and noise interference better, it needs to simulate and experiment. Firstly aiming to the blur and noise problem in degradation image, it tests and verifies the proved inverse filtering algorithm and wiener filtering respectively and compare the result effect.

Under same condition, the experiment selects two images with outstanding blur and noise to simulate (one is number plate extraction with motion and blur, the other is satellite image with large noisy). It uses the method of inverse filtering and wiener filtering for the two images to recover respectivly, defined six groups value of $K$, calculates and record the recovery image $G M G 、 L S$ to compare the quality of the restored image.

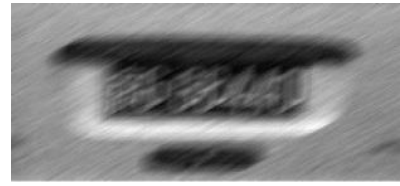

(a) Blur image

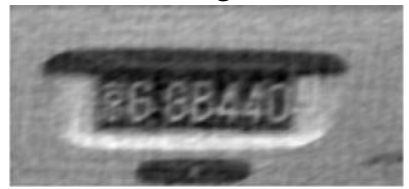

(c)Restoration image by inverse filtering

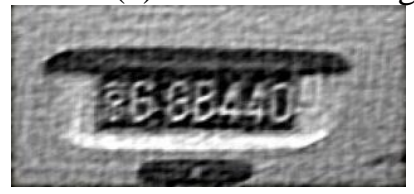

(d) Restoration image by wiener filtering $(K=0.001)$

\section{Figure 3. Restoration Image by Different Filtering Technology}

From the figures above, the blur number plate can easily to be distinguished letters on the plate. While the image worked by the inverse filtering is better than the wiener filtering, the license plate image of wiener filtering appears distorting compared with the original image. So it is better for the blurred image to take the method of inverse filtering than wiener filtering. For the satellite image with large noise, by inverse filtering algorithm the image quality is improved, but the edge of the satellite is not accurate, and 
appears multiple rings. While by wiener filtering image quality obtains good recovery, and can describe the noisy image accurately. Thus for the large noisy image, it is better to take the method of wiener filtering than inverse filtering. Therefore, inverse filtering restoration algorithm is applied in solving the problem of fuzzy image recovery algorithm, wiener filtering restoration algorithm is applied to solve the problem of noise disturbing image.

By analyzing data in table, it's known that for number plate the value of $G M G$ and $L S$ from inverse filtering are all bigger than wiener filtering. According to formula above, the bigger the value is, the higher the quality is, and the clearer the image is. It's mainly the blur problem for number plate. It shows that the image quality with inverse filter is better than wiener filter.

\section{Table 1. Image Quality Assessment under Different Restoration Algorithm}

\begin{tabular}{|c|c|c|c|}
\hline \multicolumn{4}{|c|}{ quality assessment method } \\
\hline \multicolumn{2}{|c|}{ restoration method } & GMG & $\mathrm{LS}$ \\
\hline \multicolumn{2}{|c|}{ inverse filter } & 6.5358 & 5.8505 \\
\hline \multirow{6}{*}{ wiener filter } & $K=0.0001$ & 6.4296 & 5.5536 \\
\hline & $\mathrm{K}=0.001$ & 5.1371 & 4.3657 \\
\hline & $K=0.005$ & 3.4085 & 2.2184 \\
\hline & $\mathrm{K}=0.01$ & 2.7523 & 1.4742 \\
\hline & $\mathrm{K}=0.1$ & 1.4796 & 0.3676 \\
\hline & $\mathrm{K}=1$ & 0.6514 & 0.2157 \\
\hline
\end{tabular}

Table 2. Image Quality Assessment of Satellite under Different Restoration Algorithm

\begin{tabular}{lccc}
\hline \multicolumn{3}{c}{ quality assessment method } \\
\multicolumn{1}{c}{ restoration method } & GMG & LS \\
\hline inverse filter & & 2.6748 & 2.5288 \\
& $\mathrm{~K}=0.0001$ & 6.9653 & 5.8854 \\
& $\mathrm{~K}=0.001$ & 6.0798 & 5.0132 \\
wiener filter & $\mathrm{K}=0.005$ & 5.1553 & 3.6534 \\
& $\mathrm{~K}=0.01$ & 3.7867 & 1.9845 \\
& $\mathrm{~K}=0.1$ & 1.8789 & 1.0121 \\
& $\mathrm{~K}=1$ & 0.9412 & 0.7543 \\
\hline
\end{tabular}

But for the satellite images, the smaller the $\mathrm{K}$ value is, the larger the noise interfered. The values of $G M G$ and $L S$ from wiener filtering are all bigger than inverse filtering. When the value $K$ increases towards 1 , the noise interference is very small or negligible, the recovery by the inverse filtering algorithm is better than wiener filtering algorithm. The data analysis result is in accordance with human observing. Based on the data in the table, for the mean values of GMG and LS by inverse filtering algorithm are all bigger than by inverse filtering in satellite images. Because the noise of satellite image is very big, it amplifies the noise by inverse filtering to recover image, which decrease effect. It shows that the inverse filtering algorithm is not applied to larger noise image restoration. In order to proving the conclusion, when it recovers the same image (satellite image) using inverse filtering with noise and without noise respectively, calculate GMG and LS to record in table.

Table 3. Image Quality Assessment under Inverse Filtering

\begin{tabular}{crc}
\hline assessment method & with noise & without noise \\
\hline GMG & 2.6748 & 4.8343 \\
\hline LS & 2.5288 & 4.6412 \\
\hline
\end{tabular}


Though the contrast between with noise and without noise, it turns out that it is better to get higher quality image by inverse filtering algorithm without noise than with noise. It is visible that inverse filtering algorithm is not apply to image restoration with larger noise.

Thus, the improved inverse filtering algorithm can work well for the blur problem, and the improved wiener filtering algorithm can work well for the noise problem.

Then, for the same image it works with inverse filtering, wiener filtering and combined filtering respectively, observe restoration images, compute there values of GMG and LS, and record in table, compare the data and the quality of image.

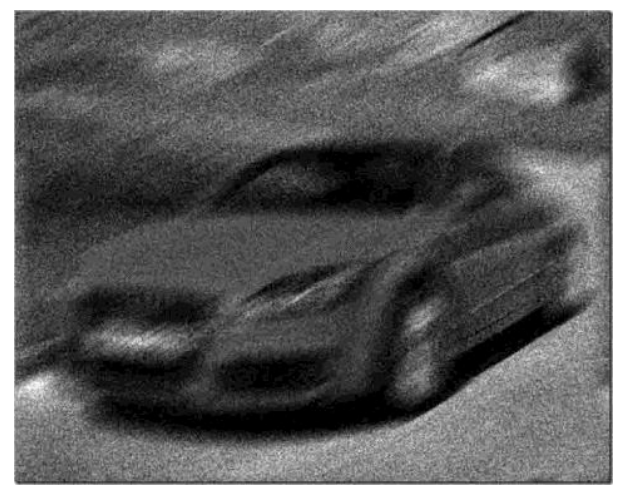

(a)True image

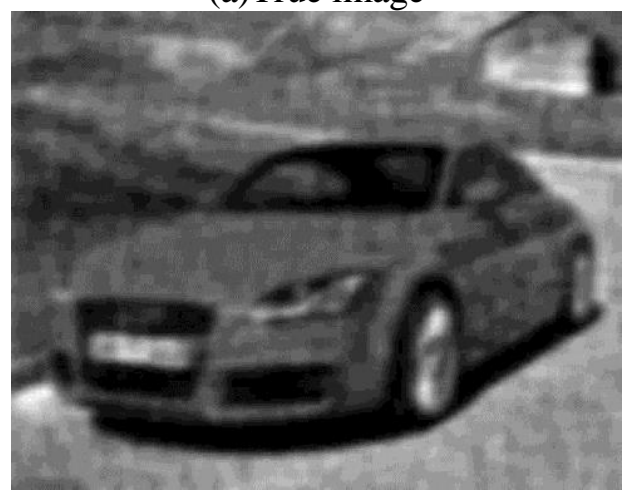

(b)Restoration image by inverse filtering

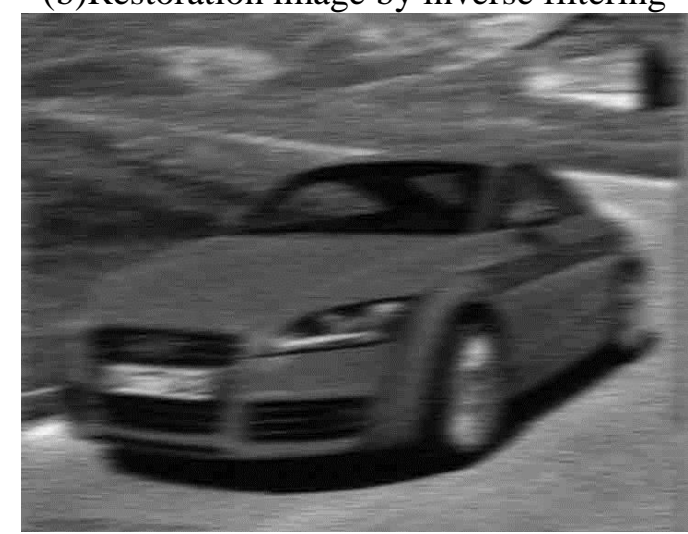

(c)Restoration image by wiener filtering 


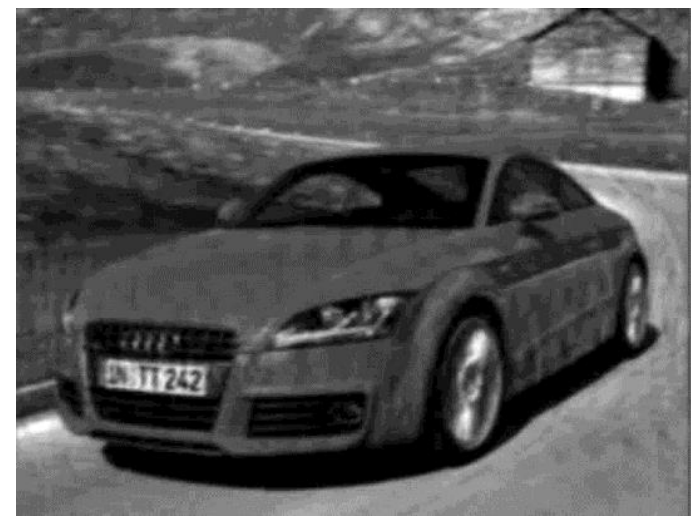

(d)Restoration image by combined filtering

\section{Figure 4. Restoration Image by Three Methods for Same Image}

Table. 4 Restoration Result by Different Algorithm

\begin{tabular}{lcc}
\hline & assessment method & \\
restoration method & GMG & LS \\
\hline inverse filtering & 4.9328 & 4.4763 \\
wiener filtering $(\mathrm{K}=0.01)$ & 5.1254 & 5.0321 \\
combined filtering & 6.8321 & 6.3211 \\
\hline
\end{tabular}

From the data above, it shows that the effect is better in combined inverse filtering and wiener filtering than others, the letters in image can be distinguished clearly. Here the effect is better by wiener filtering than inverse filtering, because the noise disturbing is very big in the image, which wiener filtering is good at working noise. But it's not good at blur problem.

It is concluded from experiment that inverse filtering is good for blur image to recover and can get better clarity; while it is not good for large noisy image, that it cannot restrain noise, and the recovery effect is poor in clarity; wiener filtering can get better restoration effect by adjusting the parameters $K$, especially for larger noisy interference, restoration effect is good; but when the $K$ value is too large, the image clarity is reduced, the contour is not sharp enough, and the effect is poor.

By using combined restoration algorithm to recover degraduation image, it's can restore image at best, and resolve the problem of blur and noise of image.

\section{Conclusion}

By analyzing the degradation reasons under motion state, the main reasons for the images quality degradation are blur and noise problems. The paper puts forward a theroy of using image restoration to solve the problem, builds a model of image restoration. Image restoration is the process of establish the image degradation model, and then use the reverse process to obtain the original image.

And it proposes a combined restoration algorithm with inverse filtering algorithm and wiener filtering algorithm to handle the blur and noise problems, explains the quality evaluation method for recovery image, and discusses objective evaluation method without reference in detail, calculates the value of GMG and LS, the bigger the value is, the better the quality is. Finally by contrasting two images related problems to demonstrate the conclusion, namely the inverse filtering restoration algorithm is applied to solve the blur problem, while the wiener filtering restoration algorithm is applied to solve the noise problem. 


\section{References}

[1] H. Zhang and D.-y. Luo, "Status and Development of Study on Blind Image Restoration Algorithm", Joural of Image and Graphics, vol. 9, no. 10, (2004), pp. 1145-1152.

[2] S.-j. Zhang, "Image projection", Beijing: Tsinghua University press, (2007).

[3] R. C. Gonzalez and R. E. Woods, "Digital Image Processing (Third Edition)", Beijing: Electronic Industry Press, (2010).

[4] Y. Yang, "The research on image restoration algorithm", Sichuan University, (2004).

[5] Q.-r. Chen, Q.-s. Lu and L.-z. Cheng, "Identification of the Motion Blurred Direction of Motion Blurred Images", Journal of National University of Defense Technology, vol. 26, no. 1, (2004), pp. 41-45.

[6] Y. Liang, "Research on high resolution image resoration algorithm based on adaptive optics compensation", Cheng Du: Photoeletric Technology Research Institute Chinese, (2006).

[7] Q. Xu, Y. Zhang and S. Xing, "Remote sensing image fusion and resolution enhancement technology", Beijing: Science Press, (2007).

[8] A. K. Katsaggelos, "Digital image restoration", Berlin, Germany: Springer-Verilog, (1991).

[9] G. R. Ayers and J.C. Dainty, "Interative Blind Deconvolution Method and Its applications", Optics Letters, vol. 13, (1988), pp. 547-549.

[10] H. C. Andrews, "Digital image restoration", Englewood Cliffs: Prentice-Hall, (1977), pp. 113-116.

[11] W.-h. Zhou, "Research on the image with Wiener Filter Algorithm", Computer Engineering and Science, vol. 21, no. 3, (2007).

[12] H. Li, Z. Lei, Z. Wang and J. Gao, "Research on Objection Information Extraction Arithmetic in Photoelectric Detection Target Base on Wavelet Analysis Method”, Przeglad Elektrotechniczny, vol. 88, no. 9, (2012), pp. 157-161.

[13] D.-f. Zhang and P.-q. Zhang, "Theoretical Analysis and Realization of Restoration of Wiener Filtering", ACTA Scientiarum Naturum Universitatis Sunyatseni, vol. 45, no. 6, (2006).

[14] M. E. Goda, "Wavelet domain image restoration and super-resolution", USA: The paper of Doctor of Philosophy in The University of Arizona, (2002).

[15] H. Li and Y. Li, "Target Damage Distribution Probability Calculation Arithmetic Based on Space Tangential Differential Unit Area”, IEEE sensors journal, vol. 15, no. 1, (2015), pp. 240-247.

[16] Fisher, Moshe and Kopeika, S. Normman, "Restoration of images degraded by motion", SPIE, vol. 1705, (1992) October, pp. 195-202.

[17] A. Nakamura, M. Kudo and A. Tanaka, "Collaborative filtering using restoration operators", PKDD (2003), LNAI 2838, pp. 339-349.

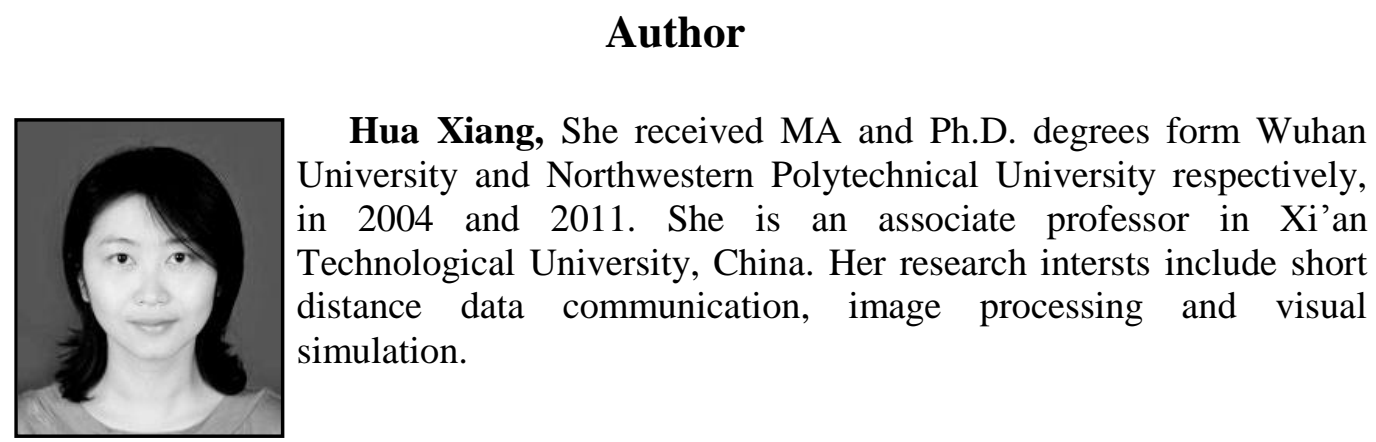

